\title{
Effects of electrical heterogeneity on transmural reentry during acute global ischemia
}

\author{
Hong Zhang ${ }^{1,2}$, Yin-Bin Jin', Zhen-Xi Zhang', Lin Yang ${ }^{3}$ and Ye-Cho Huang ${ }^{2}$ \\ ${ }^{1}$ School of Electrical Engineering, Xian Jiaotong University, Xian 710049, P. R. China; E-mail: maxr@263.net \\ 2 The Key Laboratory of Biomedical Information Engineering of Ministry of Education of China, School of Life Science and \\ Technology, Xian Jiaotong University, Xian 710049, P. R. China \\ ${ }^{3}$ Cardiology Department, The First Hospital, Xian Jiaotong University, Xian 710061, P. R. China
}

\begin{abstract}
Ventricular arrhythmias are commonly observed in patients with ischemia. It is reported that the electrophysiological changes evoked by ischemia are greater in the epicardium than in the endocardium. To investigate the effects of this heterogeneity on transmural reentry, the computer simulation method is used. A two-dimensional model which can reproduce the endocardial, epicardial and middle cell types, approximate the ischemic characteristics and distribution of the ischemic severity is developed by setting different ratios of the maximum conductance of the rapid and slow inward rectifier potassium currents and considering the three major component conditions of acute ischemia at the ionic level. The results demonstrate that action potentials of the ischemic cells have elevated resting potential, shortened duration, slowed upstroke and declined amplitude. Conduction velocity is much more depressed in the epicardium because of the ischemia-induced transmural gradient of excitability. The epicardially initiated activation has wider vulnerable window and more possibility to cause unidirectional propagation even reentry. Dispersion of the excitability is proposed to be the underlying mechanism.
\end{abstract}

Key words: Arrhythmia - Acute ischemia - Computer simulation - Reentry - Transmural heterogeneity

\section{Introduction}

It is well known that, in the ischemic myocardium, cellular architecture and heterogeneous changes in metabolic and ionic conditions interact to cause spatial and temporal heterogeneity of electrical properties leading to such lethal arrhythmias as ventricular tachycardia and ventricular fibrillation (Cascio 2001; Rubart and Zipes 2005; Lopshire and Zipes 2006). Yet, because of the complexity of the cardiac architecture, the details of their interaction, and the initiation and maintenance of reentry, remain poorly understood.

Now with modern mathematical models, the complex hypotheses can be posed and tested by the computational approach, thereby providing new insights into the fundamental mechanisms of the ischemia-related arrhythmias. The

Correspondence to: Hong Zhang, School of Electrical Engineering, Xian Jiaotong University, 28 West Xianning Road, Xian, Shaanxi 710049, P. R. China

E-mail: maxr@263.net questions of how an ion channel contributes to the states of the disease, or is altered as a byproduct of the disease itself, can be answered with this approach. The understanding of the possible mechanisms of reentrant initiation can provide the foundations for further research on the prevention and management of ventricular tachycardia and fibrillation.

Up to now most of the simulation researches have not yet taken transmural heterogeneity into consideration (Xie et al. 2001; Clayton et al. 2002; Blanca et al. 2004; Zhang et al. 2006; Trenor et al. 2007). However, it has been demonstrated that there are regional differences in electrical activities in the ventricular wall of the guinea pig, canine, and human (Akar et al. 2002; Henry et al. 2004). The endocardial ventricular cells have longer action potential durations (APDs) than the epicardial cells. A subpopulation of cells (mid-myocardial M cells) show a longer APD and a steeper dependence of APD on pacing rate than the epicardial and endocardial ventricular cell types. In addition, it is reported that acute ischemia depresses excitability more rapidly in the epicardium than in the endocardium (Wu and Zipes 2002). Due to these com- 
plex interactions, therefore, how the transmural dispersion in APD and other electrical properties change during acute global ischemia and their roles in the reentrant initiation and maintenance are only partially known today.

Our study in this paper is aimed to examine the electrical activities in the ventricular wall during global ischemia and their effects on reentrant initiation by the computer simulation method. Our investigations mainly focus on acute ischemia (first 10-12 min) before the occurrence of gap junction uncoupling and irreversible cell damage.

\section{Materials and Methods}

\section{Model selection}

Now there are several ionic mathematical models of ventricular muscle available, including the Beeler-Reuter model (Beeler and Reuter 1977), phase 1 and 2 Luo-Rudy models (Luo and Rudy 1991, 1994), and Noble models (Noble et al. 1991, 1998). They all summarize the results of voltage clamp experiments, but none of them are definitive in that they all represent steps in an ongoing process of modeling the behavior of ventricular cells. The Noble and Luo-Rudy models have described concentrations of several of the ionic species $\mathrm{Na}^{+}, \mathrm{K}^{+}$and $\mathrm{Ca}^{2+}$ that vary in time rather than stay fixed, internal $\mathrm{Ca}^{2+}$ dynamics (involving $\mathrm{Ca}^{2+}$ uptake by and release from sarcoplasmic reticulum), and $\mathrm{Na}^{+}-\mathrm{K}^{+}$ pump and $\mathrm{Na}^{+}-\mathrm{Ca}^{2+}$ exchange currents (Noble et al. 1991, 1998; Luo and Rudy 1994). These detailed models are more realistic in reproducing the characteristics of the cellular electrophysiology.

In our study we choose to use a comprehensive mathematical model reported in (Noble et al. 1991). But the delayed rectifier $\mathrm{K}^{+}$current in this model is replaced by the rapid $\left(I_{\mathrm{Kr}}\right)$ and slow $\left(I_{\mathrm{Ks}}\right)$ components described in (Noble et al. 1998). And the ATP-sensitive $\mathrm{K}^{+}$current is also incorporated into the equations to facilitate the ischemic study since it is reported that anoxia and metabolic blockade decrease concentration of ATP in the cell.

\section{Numerical solution}

The action potential of a mammalian ventricular cell can be described by the following ordinary differential equation.

$$
\frac{d V}{d t}=-\frac{1}{C_{m}}\left(I_{i o n}+I_{s t}\right)
$$

where $V$ is the transmembrane potential, $C_{m}$ is the membrane capacitance, $t$ is the time, $I_{\mathrm{st}}$ is the stimulation current and $I_{\text {ion }}$ is the total ionic current.

In this study $I_{\text {ion }}$ involves in as many as eleven currents. $I_{\text {ion }}$ $=I_{\mathrm{Na}}+I_{\mathrm{Kr}}+I_{\mathrm{Ks}}+I_{\mathrm{K}(\mathrm{ATP})}+I_{\mathrm{KNa}}+I_{\mathrm{K} 1}+I_{\mathrm{bCa}}+I_{\mathrm{CaL}}+I_{\mathrm{bK}}$
$+I_{\mathrm{bNa}}+I_{\mathrm{NaCa}}$, where $I_{\mathrm{Na}}$ is the fast sodium current; $I_{\mathrm{Kr}}$ and $I_{\mathrm{Ks}}$ are the rapid and slow delayed rectifier potassium current, respectively; $I_{\mathrm{K}(\mathrm{ATP})}$ is the ATP-sensitive potassium current; $I_{\mathrm{KNa}}$ is the $\mathrm{Na}^{+}-\mathrm{K}^{+}$exchange pump current; $I_{\mathrm{K} 1}$ is the inward rectifier potassium current; $I_{\mathrm{bCa}}$ is the background calcium current; $I_{\mathrm{CaL}}$ is the L-type calcium current; $I_{\mathrm{bK}}$ is the background potassium current; $I_{\mathrm{bNa}}$ is the background sodium current; $I_{\mathrm{NaCa}}$ is the $\mathrm{Na}^{+}-\mathrm{Ca}^{2+}$ exchange current. Details about each current can be found in Noble et al. (1991, 1998).

In general, $I_{\text {ion }}$ is a function of voltage $V$, gating variables $Y_{1}, \ldots Y_{i}, \ldots Y_{M}$, and ion concentrations $Z_{1}, \ldots Z_{i}, \ldots Z_{N}$. The gating variables $Y_{i}$ satisfy the following type of ordinary differential equation:

$$
\frac{d Y_{i}}{d t}=\alpha_{i}\left(1-Y_{i}\right)-\beta_{i} Y_{i} \quad i=1, \ldots, M
$$

where $\alpha_{i}$ are rate constants and $\beta_{i}$ are only voltage-dependent. The ion concentrations $Z_{i}$ satisfy another set of ordinary differential equation:

$$
\frac{d Z_{i}}{d t}=f_{i}\left(I_{Z_{i}}, V, Z_{i}\right) \quad i=1, \ldots, N
$$

where $I z_{i}$ is the $Z_{i}$ related ionic current.

Cardiac tissue can be treated as a continuous system connected by gap junctions between adjacent cells. Therefore, in an isotropic monodomain system, the single cell model is usually incorporated into a partial differential equation for an excitable medium in the plane $(x, y)$

$$
C_{m} \frac{\partial V}{\partial t}=-\left(I_{i o n}+I_{s t}\right)+\frac{1}{\rho_{x} S_{v}} \frac{\partial^{2} V}{\partial x^{2}}+\frac{1}{\rho_{y} S_{v}} \frac{\partial^{2} V}{\partial y^{2}}
$$

where $x$ and $y$ are the spatial coordinates in the sheet, $\rho_{x}$ and $\rho_{y}$ are the bulk cytoplasmic resistivity, and $S_{v}$ is the surfaceto-volume ratio. Here we set $\rho_{x}=\rho_{y}=0.2 \mathrm{k} \Omega \cdot \mathrm{cm}$, and $S_{v}$ $=5000 \mathrm{~cm}^{-1}$.

We use the time splitting method to integrate equations (1) to (4). The solving procedure is divided into two steps. The second-order Runge-Kutta method with adaptive time step varied from minimum time step $\left(\Delta t_{\min }=0.01 \mathrm{~ms}\right)$ to maximum time step $\left(\Delta t_{\max }=0.05 \mathrm{~ms}\right)$ is applied to solve the action potential of each single cell. To improve the numerical accuracy, the perturbation finite difference instead of the five-point centered difference method is employed to integrate the partial differential equation, in which the time step $\Delta t=0.05 \mathrm{~ms}$ is used to keep all cells synchronized. The spatial step is $\Delta x=\Delta y=h=0.01 \mathrm{~cm}$. With this approach, the relative error can decline about $2 \%$. Details of the solving procedures can be found in Zhang et al. (2006).

Numerical integration and data analysis are carried out on the computer with $2.8 \mathrm{GHz}$ processor using programs written in visual $\mathrm{C}++$ programming language. Black and white figures are plotted based on the data stored in the data files. 


\section{Transmural model development}

Recently, extensive studies have demonstrated that electrical activities of the cells isolated from the sub-epicardial and endocardial myocardium display different characteristics. Epicardial, endocardial and midmyocardial (or "M cell") regions across the ventricular wall were found (Akar et al. 2002; Henry et al. 2004). In the guinea-pig ventricle, APD is significantly shorter in the sub-epicardium than in the sub-endocardium. The difference in APD is assumed to be mainly due to the densities of $I_{\mathrm{Kr}}$ and $I_{\mathrm{Ks}}$, and their associated channel kinetics. It has been found that the transmural heterogeneity of $I_{\mathrm{Ks}}$ density plays a major role in the transmural difference of APD. $I_{\mathrm{Kr}}$ is relatively uniform across the ventricular wall, contributing much less of the heterogeneity (Viswanathan et al. 1999). Therefore, in our study the $I_{\mathrm{Kr}}$ density is kept consistent while the density of $I_{\mathrm{Ks}}$ is changed. Heterogeneity of $I_{\mathrm{Kr}}$ and $I_{\mathrm{Ks}}$ densities is introduced by altering their maximum conductance $\mathrm{G}_{\mathrm{Kr}}$ and $\mathrm{G}_{\mathrm{Ks}}$. Thus, the simulated transmural cell types are labeled with maximum conductance ratios. Because the $I_{\mathrm{Ks}}$ density is greatly reduced in the $M$ cells, thereby the conductance ratios of the epicardial, endocardial and $\mathrm{M}$ cells are set to be $\mathrm{G}_{\mathrm{Ks}}: \mathrm{G}_{\mathrm{Kr}}=23: 1,15: 1$ and $7: 1$ (Viswanathan et al. 1999), respectively. Other differences such as the presence of a larger $I_{\text {to }}$ in the epicardial cells in certain species that can affect morphology of the action potential are not considered since we mainly focus on electrical activities across the transmural wall.

A two-dimensional square tissue including $100 \times 100$ cells is developed based on the above single cell model. The electrical propagation within the whole tissue is what we mostly concerns here, not the electrical activities within a single cell, so for simplicity, we choose a square as a cell model. The size of each single cell set to be $100 \mu \mathrm{m} \times 100$ $\mu \mathrm{m}$. So the thin-sliced square section of the ventricular myocardium is $10 \mathrm{~mm} \times 10 \mathrm{~mm}$. Each cell is electrically coupled with four immediate neighbors by gap junctions. To simulate the regional differences across the transmural wall, the whole tissue is electrically divided into three simple layers with one of the three cell types located in one layer.

\section{Ischemic simulation across the transmural wall}

During ischemia, a decrease in inward currents (principally $I_{\mathrm{Na}}$ and $I_{\mathrm{Ca}}$ ) and/or an increase in outward currents (principally $\mathrm{K}^{+}$currents) predisposes to the outward shift in the balance of currents. Abrupt arrest of coronary flow to the myocardium deprives ventricular myocytes of $\mathrm{O}_{2}$ and causes the cessation of delivery of metabolites, resulting in a cascade of pathophysiological events that are associated with a decrease in inward currents of $I_{\mathrm{Na}}$ and $I_{\mathrm{Ca}}$ and a significant increase in outward currents such as $I_{\mathrm{K}}$ (ATP) (Carmeliet 1999). Therefore, to address our issue the ionic and metabolic conditions of acute ischemia that affect the cell electrophysiology are introduced as three components in our study (Shaw and Rudy 1997): 1) increase in the extracellular $\mathrm{K}^{+}$concentration $\left.\left[\mathrm{K}^{+}\right]_{0}, 2\right)$ intracellular and extracellular acidosis (decrease in $\mathrm{pH}$ ), and 3 ) anoxia and metabolic blockade. As in our previous study (Zhang et al. 2006), here the effects of acidosis are modeled through a decrease in $\mathrm{Na}^{+}$ and $\mathrm{Ca}^{2+}$ channel conductance, $\mathrm{G}_{\mathrm{Na}}$ and $\mathrm{G}_{\mathrm{CaL}}$, respectively, and a slight decline of intracellular $\mathrm{K}^{+}$concentration $\left[\mathrm{K}^{+}\right]_{\mathrm{i}}$. The anoxia is approximated by reducing the intracellular ATP concentration $[\mathrm{ATP}]_{\mathrm{i}}$ that affects the ATP-dependent potassium channels.

It has long been recognized that endocardium and epicardium have different responses to ischemia. Pathological findings suggest that the endocardium has a greater degree of myocardial necrosis, fibrosis, and metabolic abnormalities than the epicardium (Jantunen and Collan 1989; Figueredo et al. 1993). The irreversible ischemic injury occurs first in the subendocardium and progressing toward the subepicardium over time (Lowe et al. 1983). However, paradoxically, from electrophysiological point of view, more and more evidence have suggested that acute myocardial ischemia induces a greater electrical depression of ventricular epicardial versus endocardial tissues (Gilmore et al. 1980; Kimura et al. 1986; Furukawa et al. 1991; Lukas and Antzelevitch 1993; Qi et al. 2000; Wu and Zipes 2002; Michailova et al. 2007; Cordeiro et al. 2008). During acute myocardial ischemia, conduction in the endocardium is relatively preserved whilst conduction in the epicardium becomes increasingly delayed and fractionated (Kimura et al. 1986). The difference between the electrophysiological response of endocardium and epicardium is particularly puzzling. The more reduced sodium current availability in ventricular epicardium is suggested to be one of the contributors to its greater sensitivity to electrical depression (Cordeiro et al. 2008). Other studies have shown the greater epicardial sensitivity to ATP sensitive potassium channel current activation and suggested that this effect may contribute to its greater electrical vulnerability to ischemic conditions (Furukawa et al. 1991; Michailova et al. 2007). In conclusion, despite the greater susceptibility of the endocardium to metabolic effects of ischemia, the electrophysiological changes evoked in the epicardium are actually greater.

Therefore, to approximate heterogeneity of the ischemic distribution in the tissue, we linearly change five ischemiarelated physiological variables $\left(\mathrm{G}_{\mathrm{Na}}, \mathrm{G}_{\mathrm{CaL}},\left[\mathrm{K}^{+}\right]_{\mathrm{i}}, \mathrm{ATP}_{\mathrm{i}}\right.$ and $\left[\mathrm{K}^{+}\right]_{\mathrm{o}}$ ) across the transmural surface. $\mathrm{G}_{\mathrm{Na}}, \mathrm{G}_{\mathrm{CaL}}$ and $\left[\mathrm{K}^{+}\right]_{\mathrm{i}}$ are gradually decreased from $2.5 \mu \mathrm{S}, 0.25 \mu \mathrm{S}$ and $145 \mathrm{mmol} / \mathrm{l}$ to $1.4 \mu \mathrm{S}, 0.14 \mu \mathrm{S}$, and $130 \mathrm{mmol} / \mathrm{l}$, respectively. $\mathrm{ATP}_{\mathrm{i}}$ is dropped from $7.5 \mathrm{mmol} / \mathrm{l}$ to $2.5 \mathrm{mmol} / \mathrm{l}$, and $\left[\mathrm{K}^{+}\right]_{\mathrm{o}}$ is increased from $4.5 \mathrm{mmol} / \mathrm{l}$ to $12.0 \mathrm{mmol} / \mathrm{l}$. 


\section{Pacing protocols}

To compare characteristics of the action potentials, the developed tissue is periodically paced with a train of 5 current pulses $\mathrm{S} 1$. Since the activation sequence during sinus rhythm typically proceeds from the endocardium to the epicardium, in our study $\mathrm{S} 1$ is always delivered at the left down corner of the endocardium with the area of $0.5 \mathrm{~mm} \times 0.5 \mathrm{~mm}$. The basic cycle length (BCL) of the stimulation is $1000 \mathrm{~ms}$ with twice the diastolic current threshold of activation. It is $30 \mu \mathrm{A} / \mu \mathrm{F}$ in amplitude and $2 \mathrm{~ms}$ in duration in our study.

\section{Vulnerable window measurement}

The vulnerable window is determined by the extrastimulus technique. After a train of basic stimuli S1 is delivered at the left down corner of the endocardium, an early extrastimulus S2 with time step $1 \mathrm{~ms}$ is then introduced at either the endocardial or the epicardial site. We define the limits of the vulnerable window as the lowest and highest values of the S1 S2 interval for which the unidirectional propagation can be initiated. Here the unidirectional propagation refers to that conduction fails or is decremental along one direction, whereas it is successful along the opposite direction.

\section{Results}

\section{Electrical properties of the single cells}

Fig. 1 displays action potentials (a), the corresponding currents $I_{\mathrm{Kr}}(\mathrm{b})$ and $I_{\mathrm{Ks}}(\mathrm{c})$ of a single epicardial (open circle), endocardial (solid circle) and middle (solid square) cells, respectively. They are calculated and plotted based on the Eq. (1) to (3) without considering the interaction and electrical propagation described by the Eq. (4). The $\mathrm{APD}_{90}$ is measured as the difference between the activation time and the time at which the action potential is $90 \%$ recovered. To facilitate comparison, magnitudes of the two kinds of currents $I_{\mathrm{Kr}}$ and $I_{\mathrm{Ks}}$ at membrane potential $20 \mathrm{mV}$ are denoted with dotted lines. As shown in Fig. 1, to the same membrane potential $I_{\mathrm{Kr}}$ is identical, whereas $I_{\mathrm{Ks}}$ is the smallest in the $M$ cell, starting from the early phase of the action potential, and sustains a much smaller peak magnitude during the course of the action potential, leading to APD of the M cell considerably longer. Therefore, among the three different cell types, the epicardial cell has the shortest APD while the $M$ cell shows the longest. The APD difference between the $M$ cell and the endocardial cell is about $19 \mathrm{~ms}$, but there is only $8 \mathrm{~ms}$ APD difference between the endocardial and the epicardial cell.

In addition, our numerical study indicates that APDs of the three transmural cell types display different degrees of

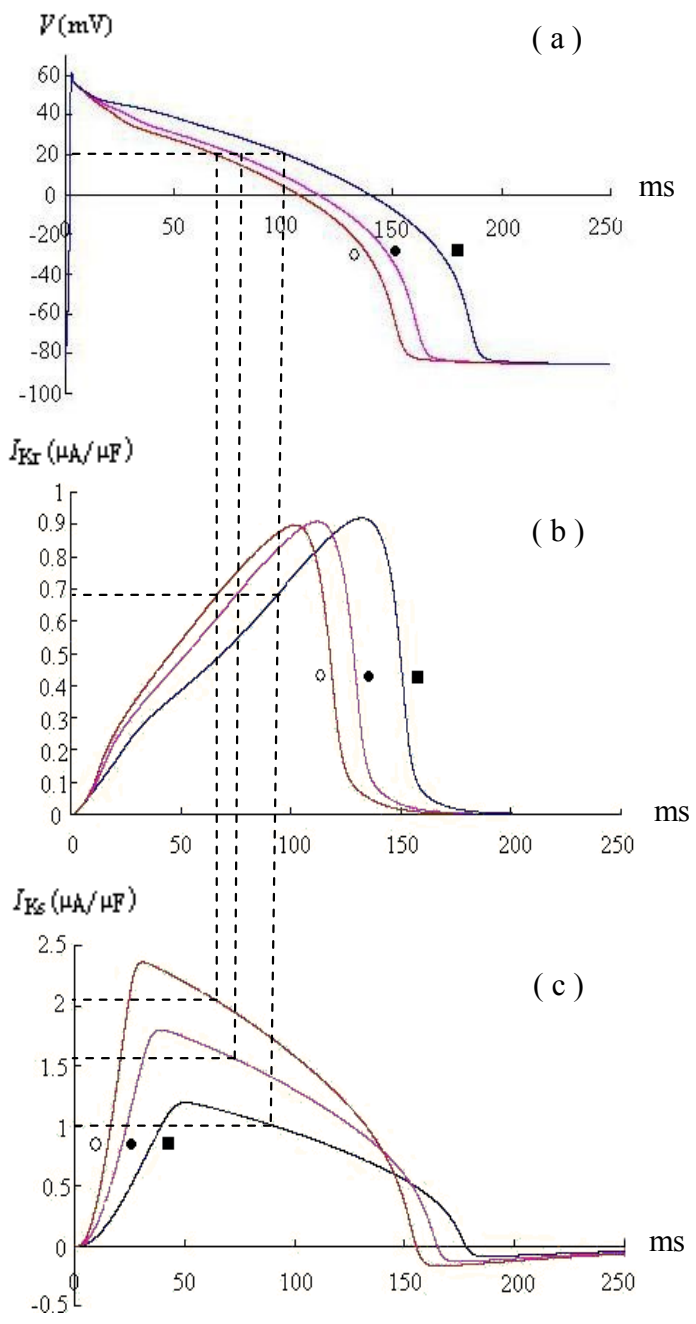

Figure 1. Action potentials (a), corresponding currents $I_{\mathrm{Kr}}(\mathrm{b})$ and $I_{\mathrm{Ks}}(\mathrm{c})$ of the isolated single cells. Three cell types (epicardial, endocardial and $\mathrm{M}$ cells) are labeled with open circle, solid circle and solid square, respectively. Dotted lines denote magnitudes of the two kinds of currents at membrane potential of $20 \mathrm{mV}$.

rate dependence. In other words, they can adjust their APDs to changes in pacing frequency with different extent. The most prominent effect of decreasing BCL is shortening of APDs. The $\mathrm{M}$ cell has much more obvious rate-dependent characteristic than the epicardial and endocardial cells. To investigate the underlying ionic mechanisms in it we examine some parameters $\left(I_{\mathrm{Kr}}, I_{\mathrm{Ks}}, I_{\mathrm{Ks}}\right.$ activation gate $X_{\mathrm{s}}$, and $\left.I_{\mathrm{NaCa}}\right)$ in the $\mathrm{M}$ cell that play the important roles during the plateau and repolarization phases of the action potential. Fig. 2 shows the results at different stimulation rate, superimposed to facilitate comparison.

$I_{\mathrm{Kr}}$ (Fig. 2b) is similar at the two pacing rates (differences secondary to APD difference should not be considered and currents should be compared at the same transmembrane 


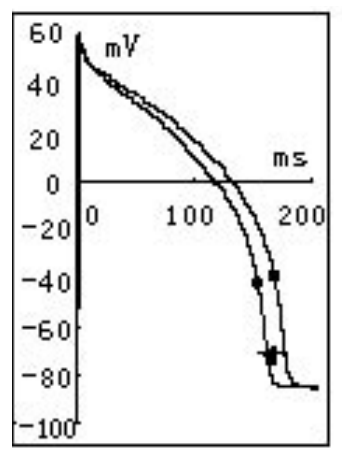

(a) AP

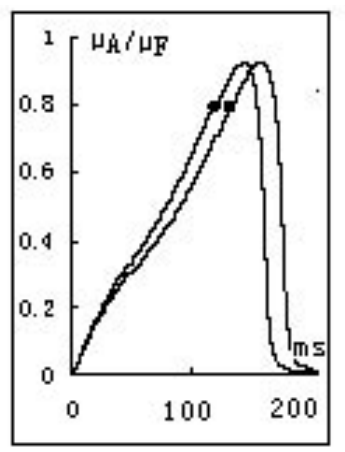

(b) $\mathrm{I}_{\mathrm{Kr}}$

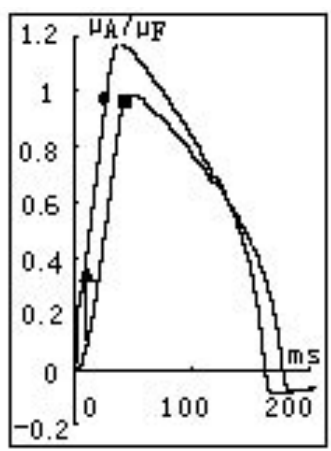

(c) $I_{K s}$

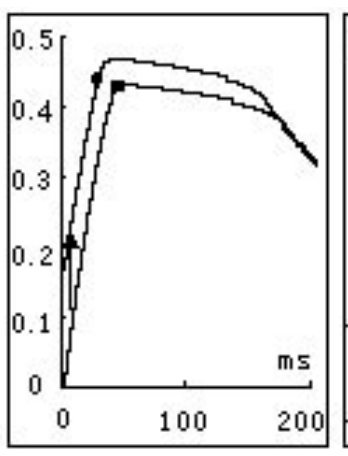

(d) $X_{s}$

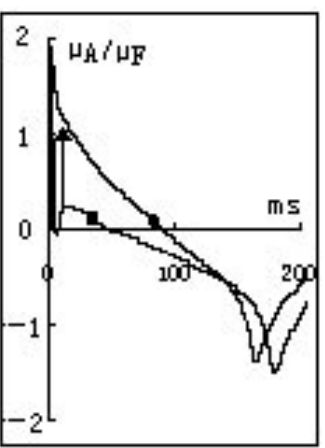

(e) $\mathrm{I}_{\mathrm{NaCa}}$

Figure 2. Underlying mechanisms of the APD adaptation to the pacing rate changes in M cells. Action potential AP (a), $I_{\mathrm{Kr}}(\mathrm{b}), I_{\mathrm{Ks}}$ (c), $I_{\mathrm{Ks}}$ activation gate $X_{\mathrm{S}}(\mathrm{d})$, and $\mathrm{Na}^{+}-\mathrm{Ca}^{2+}$ exchange current $I_{\mathrm{NaCa}}(\mathrm{e})$ are computed at the slow pacing $(\mathrm{BCL}=1000 \mathrm{~ms}$, labeled with solid square) and fast pacing $(\mathrm{BCL}=400 \mathrm{~ms}$, labeled with solid circle), respectively.

potential), resulting in less contribution to rate adaptation. But the arrow in Fig. 2c illustrates that there is greater residual activation of $I_{\mathrm{Ks}}$ for the shorter BCL at the onset of depolarization, which lead to greater repolarization current and shorter APD. Similarly, Fig. $2 \mathrm{~d}$ also shows that $I_{\mathrm{Ks}}$ activation gate $X_{\mathrm{s}}$ is larger at faster pacing, reflecting a greater degree of activation and of channel availability. This property further proves the results drawn from Fig. 2c. $I_{\mathrm{NaCa}}$ (Fig. 2e), at the short BCL, initially is a remarkably outward current during the plateau phase of the action potential, acting to shorten the APD at the shorter BCL. But it diminishes relatively quickly, reverses direction, and becomes a relatively large depolarizing current. This limits its role in APD shortening. Therefore, the accumulation of $I_{\mathrm{Ks}}$ activation caused by incomplete deactivation, together with initially increased outward current $I_{\mathrm{NaCa}}$ is implied to act to shorten the APD at the shorter BCL, which constitutes adaptation processes in the $\mathrm{M}$ cell. Our numerical experiment also shows the same underlying mechanisms for APD rate dependence in the epicardial and endocardial cells.

Because in the Noble model (1998) only one activation gate $X_{\mathrm{s}}$ is introduced instead of two $\left(X_{\mathrm{s} 1}\right.$ for slow activation; $X_{\mathrm{s} 2}$ for fast activation) in the Luo-Rudy model (Luo and Rudy 1994), it results in peak magnitude of $I_{\mathrm{Ks}}$ appearing earlier in the Noble model. Except this discrepancy, our findings and results here are in good agreement with the study in Viswanathan et al. (1999), indicating reliability of our model.

\section{Action potentials in the normal transmural tissue}

Fig. 3 displays the developed tissue, applied stimulation and some action potentials across the transmural layers after the forth stimulus is delivered. Action potentials at the sites A to $\mathrm{D}, \mathrm{E}$ to $\mathrm{H}$, and $\mathrm{I}$ to $\mathrm{L}$ belong to the endocardial, $\mathrm{M}$ and epicardial layers, respectively.
Two parallel dotted lines in Fig. 3 indicate boundary of the three cell types. The endocardial layer denoted with Endo is positioned at the left-hand area of the first dotted line. The epicardial layer denoted with Epi is at the right-hand area of the second dotted line, while the $\mathrm{M}$ cells denoted with $\mathrm{M}$ locate between these two layers. Areas of the endocardial, epicardial and M layers are (in $\mathrm{mm}$ ): $4.0 \times 10,4.0 \times 10$ and $2.0 \times 10$, respectively.

Note that in Fig. 3, since resting potential is greatly elevated, the excitation wavefront is clearly visible in light gray after the stimulus. The whole tissue is activated with electrical propagation. By comparison, we find the marked APD differences among the cells positioned in the middle of each layer, such as: the sites B, C in the endocardium; F, G in the $\mathrm{M}$ myocardium; I, J in the epicardium. The epicardial cells have the shortest APDs, while the M cells have the longest. However, compared with the action potentials of the isolated single cells in Fig. 1, APD differences are reduced among three cell types in the tissue, especially in boundary due to the electrotonic interaction and homogenizing effect of cell-to-cell coupling present in the intact tissue. Importantly, the $\mathrm{M}$ cell zone is blurred in space and boundary, thereby reducing APD dispersion across the transmural surface. Moreover, no obvious differences in the amplitude, resting potential and upstroke of the action potential are found across the ventricular wall.

\section{Transmural electrical activities during acute global ischemia}

Fig. 4 shows action potentials at the different sites on the simulated transmural ischemic tissue, and isochrone maps after the forth stimulus is delivered. The isochrone maps are plotted at $V=-20 \mathrm{mV}$ every $2 \mathrm{~ms}$ and $10 \mathrm{~ms}$ for depolarizing and repolarizing processes, respectively. 


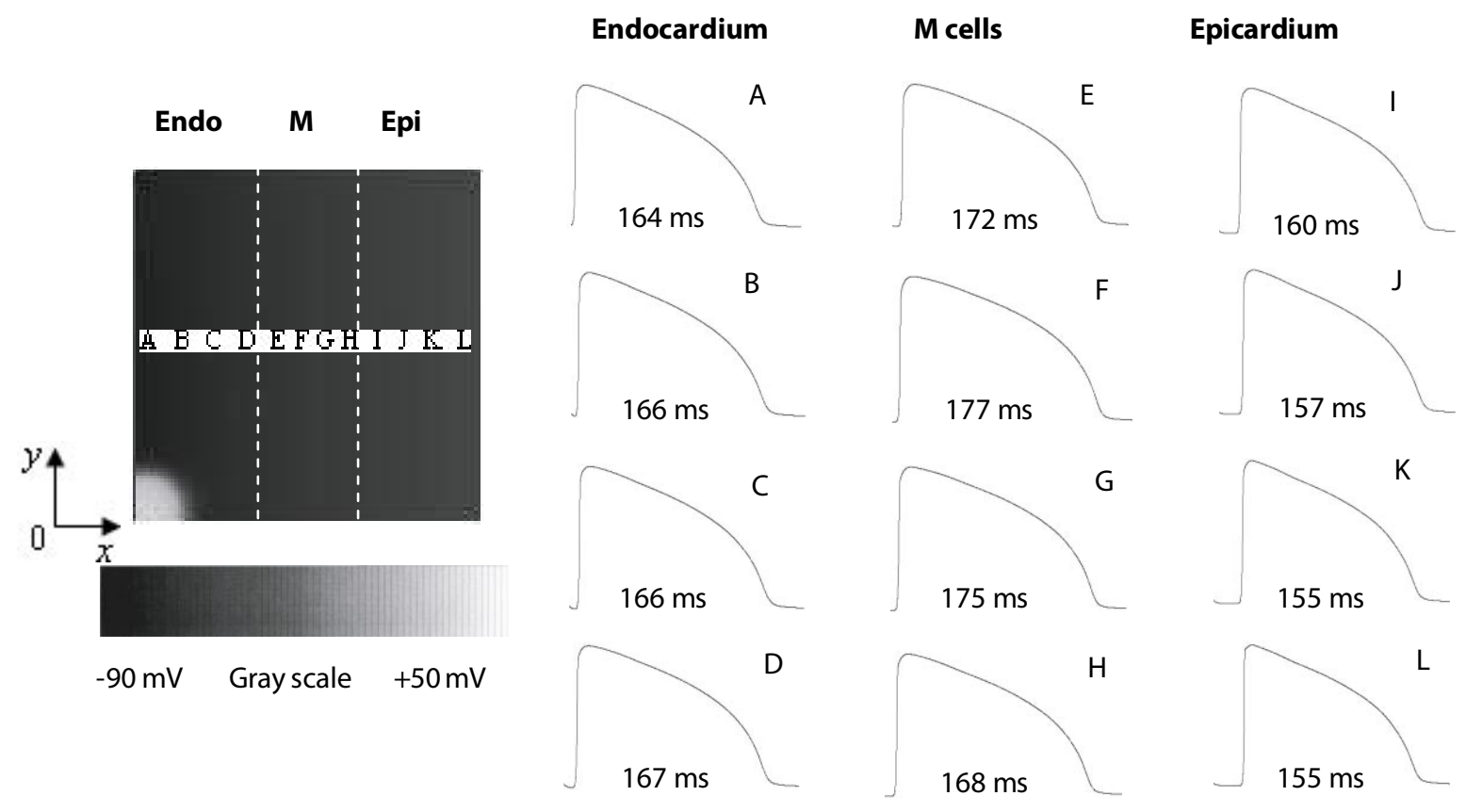

Figure 3. Developed transmural tissue, applied stimulation and action potentials. The tissue includes $100 \times 100$ cells. Two dotted lines on the tissue indicate distribution of the three cell types. The endocardial layer denoted with Endo is positioned at the left-hand area of the first dotted line. The epicardial layer denoted with Epi is sited at the right-hand area of the second dotted line, while the $\mathrm{M}$ cells denoted with $\mathrm{M}$ locate between these two layers. Displayed action potentials locate in the tissue with the following coordinates: $\mathrm{A}(10,50), \mathrm{B}(20,50)$, $\mathrm{C}(30,50), \mathrm{D}(39,50), \mathrm{E}(45,50), \mathrm{F}(50,50), \mathrm{G}(55,50), \mathrm{H}(59,50), \mathrm{I}(65,50), \mathrm{J}(75,50), \mathrm{K}(85,50), \mathrm{L}(95,50)$. Data under each action potential is its action potential duration. The gray scale bar indicates the transmembrane potential of the cells.

After inspecting, we find that with gradual ischemic severity across the transmural surface, the cells are characterized with more and more depressed APDs, much slower action potential upstroke, less negative resting potential, and smaller amplitude. Ischemia shortened APDs of all cells. But compared with Fig. 3 where the M cells have the longest APDs, in Fig. 4 APDs of the M cells are greatly shortened due to their more severe ischemia than the endocardial cells, resulting in their APDs are no longer the largest. Meanwhile, APDs of the epicardial cells reduce further due to their most ischemic severity. Therefore, in the repolarizing isochrone map (Fig. $4 \mathrm{~N}$ ) we can always find the area at the right down corner repolarizes first, next comes the $\mathrm{M}$ cells, and the lefthand side is the last part to repolarize to the resting potential. Generally speaking, in the whole ischemic tissue, APD dispersion is greatly enlarged by contrast with Fig. 3 .

Fig. $4 \mathrm{M}$ can provide detailed insight into the excitation propagation. As noted there are different isochrone densities across the ventricular wall, indicating the different propagation velocities. Across the transmural surface ischemia becomes more and more severe from the left-hand side to the right-hand side, leading to more and more densely isochrone lines, implying the slowest activation conduction in the epicardium and the fastest conduction in the endocardium.
After calculation we find propagation velocity in the whole tissue drops from 560 mm/s in Fig. 3 to 255 mm/s in Fig. 4 . According to our previous studies (Zhang et al. 2005; Yang et al. 2007), the sodium channel inactivation gate, which provides the degree of $I_{\mathrm{Na}}$ recovery and the fraction of available sodium channels, was smaller and needed a longer recovery time in the ischemic cell. With severity of ischemia, it became much less and needed a much longer recovery time, resulting in lower excitability and longer refractory period. Therefore, because of the heterogeneity of ischemic distribution there is transmural gradient of excitability in the tissue which can lead to different velocities along the electrically diffusing direction.

\section{Vulnerable window and initiation of reentry}

Today it is widely believed that the fundamental mechanism responsible for ventricular tachycardia or ventricular fibrillation is reentry. But the underlying factors that may impact reentry initiation remain incompletely unknown, especially under ischemic conditions. Here by comparing vulnerable window we intend to find which one, the epicardial or endocardial premature beat is easier to induce transmural reentry during acute global ischemia. 


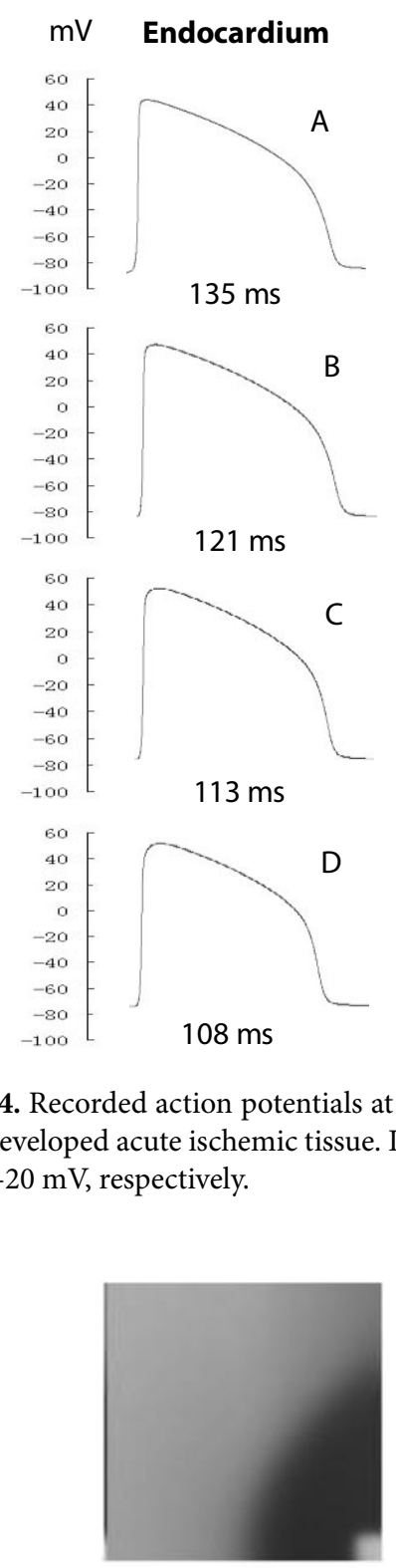

(a) $0 \mathrm{~ms}$

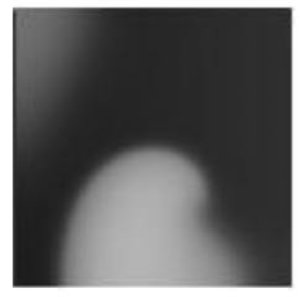

(e) $50 \mathrm{~ms}$

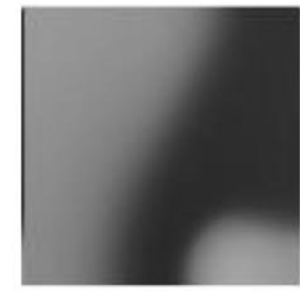

(b) $20 \mathrm{~ms}$

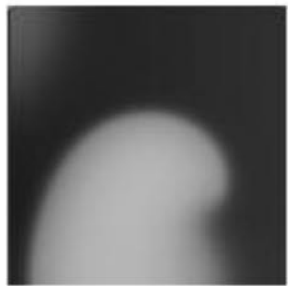

(f) $55 \mathrm{~ms}$

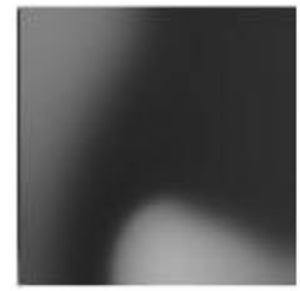

(c) $40 \mathrm{~ms}$

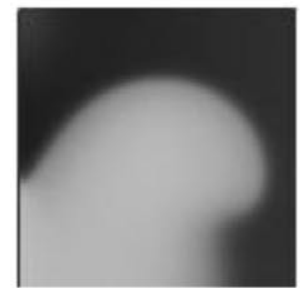

(g) $60 \mathrm{~ms}$

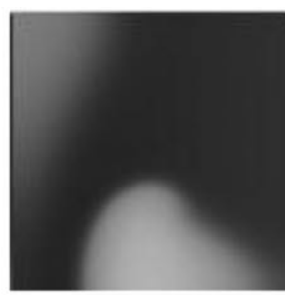

(d) $45 \mathrm{~ms}$

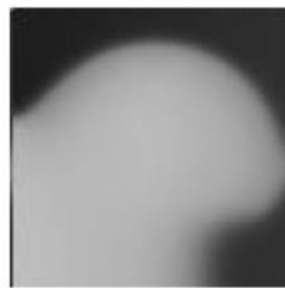

(h) $65 \mathrm{~ms}$

Figure 5. Processes of unidirectional propagation (a to c) and reentry initiation ( $\mathrm{d}$ to $\mathrm{h}$ ) after the premature stimulation S2 is delivered at the epicardium. Data under each pattern is the time after S2 is applied. Brightness represents the membrane voltage by the gray scale shown in Fig. 3. 
Fig. 5 shows an example of the processes during which a unidirectional propagation and reentry is initiated after the epicardial premature stimulation. As noted, due to the shortest APD of the epicardium, when a critically timed stimulation is delivered at the right down corner, the cells in this area have gradually repolarized from the preceding excitation. However, because of the low excitability caused by severe ischemia, the initial electrical distribution (Fig. $5 a$ and $5 b$ ) is formed due to the electrotonic interaction. Conduction velocity is very slow at the beginning, finally leading to a unidirectional propagation (Fig. 5c). However, the reduced sensitivity of the middle and endocardial layer conduction to ischemia can cause the epicardially initiated activation to conduct rapidly along these two regions and finally forms the tip of the reentry (Fig. 5d and $5 \mathrm{e})$. Then the activation reenters the epicardial myocardium and makes it activate again (Fig. $5 \mathrm{f}$ to $5 \mathrm{~h}$ ).

If the premature stimulation is outside the vulnerable window, no unidirectional propagation can be induced. As shown in Fig. 6, when the premature stimulation is delivered later than the highest values of the S1-S2 interval, the excitation can activate the whole tissue with fast conduction velocity due to their recovered excitability. Contrarily, if the premature stimulation is delivered so early that the tissue is still in refractory period, the activation can't spread and finally vanishes (Fig. 7).

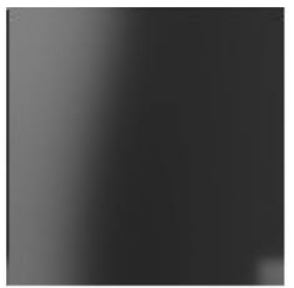

(a) $0 \mathrm{~ms}$

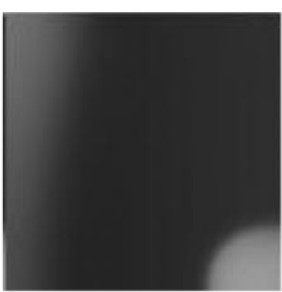

(b) $10 \mathrm{~ms}$

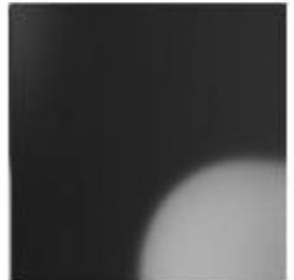

(c) $20 \mathrm{~ms}$

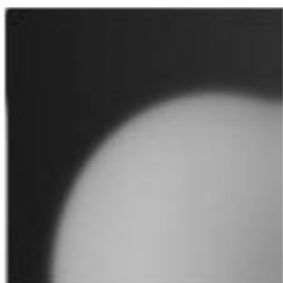

(d) $30 \mathrm{~ms}$

Figure 6. Processes of two-directional propagation if the premature stimulation S2 is delivered later than the highest limit of the vulnerable window. Data under each pattern is the time after S2 is applied.

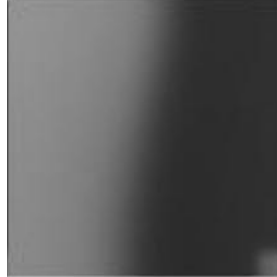

(a) $0 \mathrm{~ms}$

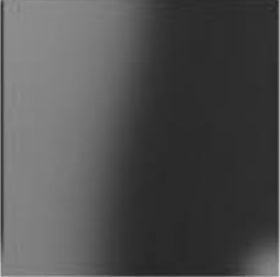

(b) $6 \mathrm{~ms}$

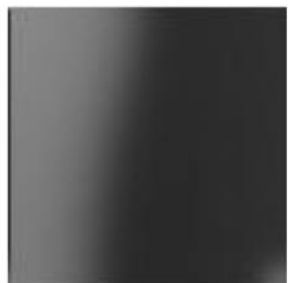

(c) $10 \mathrm{~ms}$

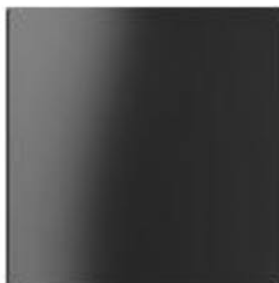

(d) $14 \mathrm{~ms}$

Figure 7. Processes of propagation block when premature stimulation S2 is delivered earlier than the lowest limit of the vulnerable window. Data under each pattern is the time just after S2 is applied. 
In our simulated two-dimensional tissue the $\mathrm{M}$ cells have the longest APDs while APDs of the epicardial cells are the shortest, although the differences between them decline in contrast to the isolated single cell due to spatially homogenizing effect. Moreover, it is demonstrated that APDs of the three cell types have rate dependence because of the accumulation of $I_{\mathrm{Ks}}$ activation and initially increased outward current $I_{\mathrm{NaCa}}$ that act to shorten the APD at the shorter BCL. The above results illustrate our developed model is capable of approximating the reality.

To simulate the ischemia within the first 10-12 min after coronary obstruction, five parameters responsible for the majority electrophysiological changes in action potential morphology and propagation are linearly altered: decrease $[\mathrm{ATP}]_{\mathrm{i}}$ for $I_{\mathrm{K}(\mathrm{ATP})}$ activation, increase $\left[\mathrm{K}^{+}\right]_{\mathrm{o}}$, and decrease maximum conductance of $I_{\mathrm{Na}}$ and $I_{\mathrm{CaL}}$, and intercellular potassium concentration $\left[\mathrm{K}^{+}\right]_{\mathrm{i}}$. By changing the above variables across the transmural surface, different severities of ischemia are taken into consideration. Based on the electrophysiological results the epicardium suffers more changes of ischemia than the endocardium, so the epicardial excitability is depressed more rapidly and severely than excitability of the endocardium. Therefore, by comparing we find some properties of the ischemic cells. These include the elevated resting potential, shortened APD, slowed upstroke and declined amplitude of the action potential. APDs of the $\mathrm{M}$ cells are no longer the largest. But APD dispersion of the whole tissue is enlarged.

As indicated, we find the electrical excitation conducts with different velocities across the transmural tissue during acute global ischemia. Propagation is much faster in the endocardium because of its less ischemic sensitivity and higher excitability. When the extrastimulus S2 is delivered at the endocardium, excitation can either rapidly spread towards the epicardium or completely blocked due to refractory period of the $\mathrm{M}$ and epicardial cells. No vulnerable window can be detected in this case. However, if S2 locates at the epicardium and critically timed, the unidirectional propagation can be induced. The lower excitability of the epicardium can not cause activation to conduct within this layer, but to spread much slowly under the electrotonic interaction. If transmural dispersion of the excitability is applicable, the activation front can then penetrate the still-viable middle and even the endocardial layers, and finally form the reentry. The faster rate of depression of excitability in the epicardium also can create an opportunity for reentrant conduction from the middle and endocardial layers back to the epicardium. In contrast, this transmural dispersion of excitability can possibly result in endocardially initiated excitation to conduct rapidly across the endocardium, but slowly moving to the epicardium without unidirectional block and thereby no reentry. The results imply that the epicardial premature beat is much easier to elicit ventricular tachycardia during acute global ischemia. Ischemia-induced transmural dispersion of excitability is proposed to be the underlying mechanism.

Vulnerable window is an effective index to evaluate the increased risk for unidirectional conduction block. Therefore, the factors increasing the vulnerable window can lead to the increase of the incidence of malignant arrhythmias. Therefore, the results give us a clinical implication that if we increase the cellular excitability, reducing its dispersion across the ventricular wall, the reentrant circuit can be prevented.

The report in Qu et al. (2006) predicts that a single endocardial extrasystole arising from the endocardium needs much lower critical gradient than a single extrasystole originating from the epicardium, implying a premature in the endocardium is easier to elicit a unidirectional propagation. This discovery seems contrary to ours. However, it should be noted that their study is accomplished under the normal conditions in which APD gradient is usually greater between the epicardium and the M myocardium than it is between the endocardium and the M myocardium. Meanwhile, dispersion of the excitability across the transmural wall is much less obvious in the healthy tissue. Our simulation is done uniquely considering the acute global ischemia in which the electrophysiological characteristics have been changed. Lower excitability in the epicardium results in longer period of post-repolarization refractoriness which can facilitate formation of the unidirectional conduction (Zhang et al. 2006). Our findings in this paper are in good agreement with the reports in Wu and Zipes (2002) in which they map the canine ventricular muscle and observe that the epicardial stimulation is easier to induce reentry than the endocardial premature beat during ischemia.

\section{Limitations of the Study}

Owing to the variable sensitivity of epicardium and endocardium to acute ischemia, spatial dispersion of electrophysiological properties is created in our model. However, the model is still relatively simple. Some factors such as the disruption of $\mathrm{Na}^{+}-\mathrm{K}^{+}$pump due to lack of ATP and subsequent decrease in $\mathrm{Na}^{+}-\mathrm{Ca}^{2+}$ exchanger due to reduced $\mathrm{Na}^{+}$ gradient are not considered. Moreover, different presence of the transient outward current $I_{\text {to }}$ is not involved in our model, although it is suggested that the presence of $I_{\text {to }}$ in the epicardium (but not in the endocardium) in some species may amplify ischemia-induced changes in other currents, even if $I_{\text {to }}$ itself is not directly affected, and the role of $I_{\text {to }}$ in ischemia-induced shortening of APD in the epicardium is proposed as well (Lukas and Antzelevitch 1993). In addition, the roles of $I_{\mathrm{K} 1}$ channels in cardiac hypoxia are just beginning to emerge recently. During hypoxia $I_{\mathrm{K} 1}$ is reported to underlie the observed early APD shortening before $\mathrm{K}_{\mathrm{ATP}}$ 
channel is activated (Piao et al. 2007). However, although acute myocardial ischemia is associated with a variety of complicated electrophysiological changes, some simulations only take accumulation of the $\left[\mathrm{K}^{+}\right]_{\mathrm{o}}$ as a single most important factor implicated in the development of ventricular fibrillation (Xu and Guevara 1998; Xie et al. 2001). We also find that although APD is remarkably impacted by the ATP depletion, $\left[\mathrm{K}^{+}\right]_{0}$ elevation has the greatest effects on the resting membrane potential depolarization, membrane excitability and conduction velocity reduction, and excitability recovery lengthening. Therefore, our results are not expected to be influenced by ignorance of the above factors.

For reentry to occur, it is necessary that an area of tissue show unidirectional block but is able to conduct an impulse in the reverse direction. Because loop arrangements are abundant in peripheral regions of the conducting system where Purkinje fibers arborize into many branches before interconnecting with muscular fibers, reentry may be easily induced around these areas if the reentrant conditions are satisfied (Elharrar and Zipes 1977). However, because Purkinje and ventricular myocytes are electrically isolated from each other except at Purkinje-ventricular junctions (Aslanidi et al. 2007), effects of Purkinje are not taken into account in our study in that what we mostly concerns in this paper is the effects of ischemic heterogeneous distribution in ventricles on electrical vulnerability, not the conduction system through the Purkinje fiber network to the ventricles. In addition, in our tissue model we don't involve in the cardiac interstitium (the space between the cardiomyocytes, containing fibroblasts, blood vessels, lymphatic vessels, adrenergic nerve endings), because our investigations mainly focus on electrical activities among myocytes through gap junctions and the acute ischemia before irreversible cell damages occur, so the role of interstitium in remodeling during myocardial infarction or heart failure (Aslanidi et al. 2007) is not obvious in our study.

It is indicated that $\mathrm{M}$ cells are not evenly distributed across the transmural wall, they may extend to the endocardial or epicardial layer (Akar et al. 2002). So the real tissue has much more complicated structure than the tissue we develop here. And our simulation is just confined to the two-dimensional tissue with one cell communicating with other four neighboring cells. If six neighborhoods are taken into account instead of four (overlapping like bricks), due to electrotonic interactions, dispersion of the APD, conduction velocity and excitability may be changed. But still the epicardium is expected to have more depressed excitability and more vulnerability for reentry.

In addition, due to the preferential prolongation of $M$ cells, they facilitate occurrence of early afterdepolarization (EAD) and triggered activities, especially in long-QT syndrome (Antzelevitch 2005). But remarkably little (if any) information is available in the literature on the $\mathrm{EAD}$ as the triggered activities for arrhythmia during ischemia, although there is a report of phase $3 \mathrm{EAD}$ in isolated rabbit hearts with activated $\mathrm{K}_{\mathrm{ATP}}$ channel (Tang et al. 2008). So we don't pay much attention to $\mathrm{EAD}$ in this paper.

However, considering the electrophysiological detail and the realistic ischemic representation, a three-dimensional setting and more realistic model should be expected in our future work.

Acknowledgments. This study is supported by the National Natural Science Foundation of P. R. China (No. 30870659 and 30971221) and the Health Foundation of Shaanxi Province, P. R. China (No. 08D23). We also thank Dr. Shien-Fong Lin (Krannert Institute of Cardiology, Indiana University School of Medicine, USA) for his advice and help.

\section{References}

Akar F. G., Yan G. X., Antzelevitch C., Rosenbaum D. S. (2002): Unique topographical distribution of $\mathrm{M}$ cells underlies reentrant mechanism of Torsad de Pointes in the Long QT syndrome. Circulation 105, 1247-1253; doi:10.1161/ hc1002.105231

Antzelevitch C. (2005): Cardiac repolarization. The long and short of it. Europace 7, S3-S9; doi:10.1016/j.eupc.2005.05.010

Aslanidi O. V., Sleiman R. N., Williamson H., Boyett M. R., Zhang H. (2007): Modelling conduction through the Purkinje ventricular junction and the short-QT syndrome associated with HERG mutation in the rabbit ventricles. Computer in cardiology 34, 241-244; doi:10.1109/ CIC.2007.4745466

Beeler G. W., Reuter H. (1977): Reconstruction of the action potential of ventricular myocardial fibres. J. Physiol. (Lond.) 268, 177-210

Blanca R., Brock M. T., James C. E., Felipe A. (2004): Effect of acute global ischemia on the upper limit of vulnerability: a simulation study. Am. J. Physiol. Heart Circ. Physiol. 286, H2078-2088; doi:10.1152/ajpheart.01175.2003

Carmeliet E. (1999): Cardiac ionic currents and acute ischemia: from channels to arrhythmias. Physiol. Rev. 79, 917-1017

Cascio W. E. (2001): Myocardial ischemia: what factors determine arrhythmogenesis? J. Cardiovasc. Electrophysiol. 12, 726-729; doi:10.1046/j.1540-8167.2001.00726.x

Clayton R. H., Parkinson K., Holden A. V. (2002): Re-entry in computational models of ischaemic myocardium. Chaos Solitons Fractals 13, 1671-1683; doi:10.1016/S09600779(01)00174-6

Cordeiro J. M., Mazza M., Goodrow R., Ulahannan N., Antzelevitch C., Di Diego J. M. (2008): Functionally distinct sodium channels in ventricular epicardial and endocardial cells contribute to a greater sensitivity of the epicardium to electrical depression. Am. J. Physiol. Heart Circ. Physiol. 295, H154-162; doi:10.1152/ajpheart.01327.2007

Elharrar V., Zipes D. P. (1977): Cardiac electrophysiologic alterations during myocardial ischemia. Am. J. Physiol. Heart Circ. Physiol. 233, H329-345 
Figueredo V. M., Brandes R., Weiner M. W., Massie B. M., Camacho S. A. (1993): Endocardial versus epicardial differences of intracellular free calcium under normal and ischemic conditions in perfused rat hearts. Circ. Res. 72, 1082-1090

Furukawa T., Kimura S., Furukawa N., Bassett A. L., Myerburg R. J. (1991): Role of cardiac ATP-regulated potassium channels in differential responses of endocardial and epicardial cells to ischemia. Circ. Res. 68, 1693-1702

Gilmore R. F., Zipes D. P. (1980): Different electrophysiological responses of canine endocardium and epicardium to combined hyperkalemia, hypoxia, and acidosis. Circ. Res. 46, 814-825

Henry H., Rappel W. J. (2004): The role of M cells and the long QT syndrome in cardiac arrhythmias: simulation studies of reentrant excitations using a detailed electrophysiological model. Chaos 14, 172-182; doi:10.1063/1.1636272

Jantunen E., Collan Y. (1989): Transmural differences in ischaemic heart disease: a quantitative histologic study. Appl. Pathol. 7, 179-187

Kimura S. H., Basset A. L., Kohya T., Kozlovskis P. L., Myerburg R. J. (1986): Simultaneous recording of action potentials from endocardium and epicardium during ischemia in the isolated cat ventricle: relation of temporal electrophysiological heterogeneities to arrhythmias. Circulation 74, 401-409

Lopshire J. C., Zipes D. P. (2006): Sudden cardiac death: better understanding of risks, mechanisms, and treatment. Circulation 114, 1134-1136; doi:10.1161/CIRCULATI ONAHA.106.647933

Lowe J. E., Cummings R. G., Adams D. H., Hull-Ryde E. A. (1983): Evidence that ischemic cell death begins in the subendocardium independent of variations in collateral flow or wall tension. Circ. 68, 190-202

Lukas A., Antzelevitch C. (1993): Differences in the electrophysiological response of canine ventricular epicardium and endocardium to ischemia. Role of the transient outward current. Circulation 88, 2903-2915

Luo C. H., Rudy Y. (1991): A model of the ventricular cardiac action potential. Depolarization, repolarization, and their interaction. Circ. Res. 68, 1501-1526

Luo C. H., Rudy Y. (1994): A dynamic model of the cardiac ventricular action potential. I. Simulations of ionic currents and concentration changes. Circ. Res. 74, 1071-1096

Michailova A., Lorentz W., McCulloch A. (2007): Modeling transmural heterogeneity of K(ATP) current in rabbit ventricular myocytes. Am. J. Physiol. Cell Physiol. 293, C542-557; doi:10.1152/ajpcell.00148.2006

Noble D., Noble S. J., Bett G. C. L. (1991): The role of sodiumcalcium exchange during the cardiac action potential. Ann. N. Y. Acad. Sci. 639, 334-353; doi:10.1111/j.17496632.1991.tb17323.x

Noble D., Varghese A., Kohl P., Noble P. (1998): Improved guineapig ventricular cell model incorporating a diadic space, Ikr and Iks, and length- and tension-dependent processes. Can. J. Cardiol. 14, 123-134

Piao L., Li J., McLerie M., Lopatin A. N. (2007): Cardiac IK1 underlies early action potential shortening during hy- poxia in the mouse heart. J. Mol. Cell. Cardiol. 43, 27-38; doi:10.1016/j.yjmcc.2007.04.002

Qi X. Y., Shi W. B., Wang H. H., Zhang Z. X., Xu Y. Q. (2000): A study on the electrophysiological heterogeneity of rabbit ventricular myocytes - the effect of ischemia on action potentials and potassium currents. Acta. Physiol. Sinica 52, 360-364

Qu Z. L., Garfinkel A., Weiss J. N. (2006): Vulnerable window for conduction block in a one-dimensional cable of cardiac cells, 1: single extrasystoles. Biophys. J. 91, 793-804; doi:10.1529/biophysj.106.080945

Rubart M., Zipes D. P. (2005): Mechanisms of sudden cardiac death. J. Clin. Invest. 115, 2305-2315; doi:10.1172/JCI26381

Shaw R. M., Rudy Y. (1997): Electrophysiologic effects of acute myocardial ischemia: a theoretical study of altered cell excitability and action potential duration. Cardiovasc. Res. 35, 256-272; doi:10.1016/S0008-6363(97)00093-X

Tang L., Joung B., Song J., Ogawa M., Chen P. S., Lin S. F. (2008): Intracellular calcium mediated late-phase 3 early afterdepolarization induces ventricular fibrillation in isolated rabbit hearts with activated KATP channel. Circulation 118, S527-528

Trenor B., Romero L., Ferrero J. M. (2007): Vulnerability to reentry in a regionally ischemic tissue: a simulation study. Ann. Biomed. Eng. 35, 1756-1770; doi:10.1007/s10439-0079353-3

Viswanathan P. C., Shaw R. M., Rudy Y. (1999): Effects of IKr and IKs heterogeneity on action potential duration and its rate dependence: a simulation study. Circulation 99, 2466-2474

Wu J., Zipes D. P. (2002): Transmural reentry triggered by epicardial stimulation during acute ischemia in canine ventricular muscle. Am. J. Physiol. Heart Circ. Physiol. 283, H2004-2011

Xie F., Qu Z., Garfinkel A., Weiss J. N. (2001): Effects of simulated ischemia on spiral wave stability. Am. J. Physiol. Heart Circ. Physiol. 280, H1667-1673

Xu A., Guevara M. R. (1998): Two forms of spiral-wave reentry in an ionic model of ischemic ventricular myocardium. Chaos 8, 157-174; doi:10.1063/1.166286

Yang Z., Zhang H., Kong S., Yue X. F., Jin J., Huang Y. C. (2007): Study for relevance of the acute myocardial ischemia to arrhythmia by optical mapping method. Physiol. Meas. 28, 481-488; doi:10.1088/0967-3334/28/5/003

Zhang H., Yang L., Jin Y. B., Zhang Z. X., Huang Y. Z. (2005): Computer simulation study of the reentrant cardiac arrhythmias in ischemic myocardium. Chin. J. Physiol. 48, $155-159$

Zhang H., Zhang Z. X., Yang L., Jin Y. B., Huang Y. Z. (2006): Mechanisms of the acute ischaemia-induced arrhythmogenesis--a simulation study. Math. Biosci. 203, 1-18; doi:10.1016/j.mbs.2006.06.003

Received: March 24, 2009

Final version accepted: July 27, 2009 